\title{
Efeitos da pressão expiratória final positiva no equilíbrio hemodinâmico e autonômico cardíaco de pacientes em ventilação mecânica invasiva: ensaio clínico
}

Effects of positive end-expiratory pressure on cardiac hemodynamic and autonomic balance in patients on invasive mechanical ventilation: a clinical trial

Efectos de la presión positiva al final de la espiración sobre el equilibrio hemodinámico y autónomo cardíaco en pacientes con ventilación mecánica invasiva: un ensayo clínico

\author{
Diego dos Passos Santiago \\ ORCID: https://orcid.org/0000-0002-0000-3362 \\ Universidade Federal de Sergipe, Brasil \\ E-mail: diegosanttiago@hotmail.com \\ Telma Cristina Fontes Cerqueira \\ ORCID: https://orcid.org/0000-0003-0488-6112 \\ Universidade Federal de Sergipe, Brasil \\ E-mail: telmac@gmail.com \\ Lays Katharinne Calheiros de Oliveira \\ ORCID: https://orcid.org/0000-0002-3092-8673 \\ Universidade Federal de Sergipe, Brasil \\ E-mail: layscalheiros91@gmail.com \\ Gabriel Cardoso Santos \\ ORCID: https://orcid.org/0000-0003-3707-8509 \\ Universidade Federal de Sergipe, Brasil \\ E-mail: cstosgabriel@gmail.com \\ Leila Fernanda Oliveira de Jesus Cardoso \\ ORCID: https://orcid.org/0000-0001-7189-0360 \\ Universidade Federal de Sergipe, Brasil \\ E-mail: leila.cardoso31@gmail.com \\ Thais Costa Mendonça \\ ORCID: https://orcid.org/0000-0002-1842-443X \\ Universidade Federal de Sergipe, Brasil \\ E-mail: thaiscostaa13@hotmail.com \\ Milena Rabelo de Souza \\ ORCID: https://orcid.org/0000-0003-3005-8070 \\ Universidade Federal de Sergipe, Brasil \\ E-mail: milenarabelo32@gmail.com \\ Anderson Passos Pinto \\ ORCID: https://orcid.org/0000-0003-4343-1882 \\ Universidade Federal de Sergipe, Brasil \\ E-mail: andersonpassospinto@hotmail.com \\ Rafaela de Siqueira Oliveira \\ ORCID: https://orcid.org/0000-0001-8310-9340 \\ Universidade Federal de Sergipe, Brasil \\ E-mail: rafaela-oliveira123@hotmail.com \\ André Sales Barreto \\ ORCID: https://orcid.org/0000-0003-3183-0966 \\ Universidade Federal de Sergipe, Brasil \\ E-mail: absfisio@ hotmail.com
}

\begin{abstract}
Resumo
Objetivo: avaliar o efeito do incremento da pressão positiva expiratória final (PEEP) sobre a hemodinâmica e balanço autonômico cardíaco de pacientes em uso de ventilação mecânica invasiva (VMI). Metodologia: Este é um estudo não probabilístico, onde os pacientes são controles deles próprios, em doentes críticos internados após 24 horas, submetidos a níveis crescentes de PEEP $\left(8,12\right.$ e $\left.15 \mathrm{cmH}_{2} \mathrm{O}\right) 15 \mathrm{mim}$ por incremento. Resultados: Trinta pacientes foram incluídos no estudo. Nenhuma alteração foi encontrada nas variáveis hemodinâmicas e de balanço autonômico cardíaco entre os pacientes que foram submetidos a níveis crescentes de PEEP $\left(8,12\right.$ e $\left.15 \mathrm{cmH}_{2} \mathrm{O}\right)$. Conclusão: No presente estudo, os níveis de PEEP 8, 12 e $15 \mathrm{cmH}_{2} \mathrm{O}$, não promoveu alterações nas respostas hemodinâmicas e balanço autonômico cardíaco em pacientes sobre uso de ventilação mecânica invasiva.
\end{abstract}


Palavras-chave: Respiração artificial; Respiração por pressão intrínseca positiva; Frequência cardíaca; Sistema nervoso.

\begin{abstract}
Objective: To evaluate the effect of increasing positive end-expiratory pressure (PEEP) on hemodynamics and cardiac autonomic balance in patients using invasive mechanical ventilation (IMV). Methodology: This is a non-probabilistic study, where patients are their own controls, in critically ill patients hospitalized after 24 hours, submitted to increasing levels of PEEP (8, 12 and $15 \mathrm{cmH} 2 \mathrm{O}) 15$ min per increment. Results: Thirty patients were included in the study. No changes were found in hemodynamic variables and cardiac autonomic balance among patients who underwent increasing levels of PEEP (8, 12 and $15 \mathrm{cmH} 2 \mathrm{O})$. Conclusion: In the present study, PEEP levels of 8,12 and $15 \mathrm{cmH} 2 \mathrm{O}$ did not promote changes in hemodynamic responses and cardiac autonomic balance in patients on invasive mechanical ventilation.
\end{abstract}

Keywords: Artificial breathing; Positive intrinsic pressure breathing; Heart rate; Nervous system.

\title{
Resumen
}

Objetivo: Evaluar el efecto del aumento de la presión positiva al final de la espiración (PEEP) sobre la hemodinámica y el equilibrio autonómico cardíaco en pacientes que utilizan ventilación mecánica invasiva (VMI). Metodología: Se trata de un estudio no probabilístico, donde los pacientes son sus propios controles, en pacientes críticos hospitalizados a las 24 horas, sometidos a niveles crecientes de PEEP (8, 12 y $15 \mathrm{cmH} 2 \mathrm{O}) 15$ min por incremento. Resultados: Se incluyeron 30 pacientes en el estudio. No se encontraron cambios en las variables hemodinámicas y el equilibrio autonómico cardíaco entre los pacientes sometidos a niveles crecientes de PEEP $(8,12$ y $15 \mathrm{cmH} 2 \mathrm{O})$. Conclusión: En el presente estudio, los niveles de PEEP de 8, 12 y $15 \mathrm{cmH} 2 \mathrm{O}$ no promovieron cambios en las respuestas hemodinámicas y el equilibrio cardíaco autónomo en pacientes con ventilación mecánica invasiva.

Palabras clave: Respiración artificial; Respiración con presión intrínseca positiva; Frecuencia cardiaca; Sistema nervioso.

\section{Introdução}

A ventilaçaõ mecânica (VM) pode substituir total ou parcialmente a respiração espontânea, promovendo uma melhor qualidade das trocas gasosas e consequentemente a diminuição do trabalho respiratório, aumentar os níveis de oxigenação, diminuir a hipercapnia e acidose respiratória e por fim, permitir a melhora da relação ventilação/perfusão (V/Q) pulmonar (Araújo et al., 2020; Machado, Eder, Dulius \& Baldisserotto, 2018). Esta, ainda pode ser classificada de duas formas, sendo ela Ventilação Mecânica não Invasiva (VNI) que acontece através de máscaras faciais conectadas ao paciente e a Ventilação Mecânica Invasiva (VMI) que é feito através da conexão do paciente com tubo endotraqueal ou traqueostomia (Machado, Eder, Dulius \& Baldisserotto, 2018).

Para a aproximação da máquina respiratória e melhor conforto do paciente, existem parâmetros ajustáveis no ventilador mecânico, a qual destacamos a Pressão Positiva Expiratória Final (PEEP) (Forgiarini, Rezende \& Forgiarini, 2013; Akker, Egal \& Groeneveld, 2013; Wang et al., 2016). Através do ventilador mecânico, a PEEP além de parâmetro, também pode ser usada como técnica de recrutamento ou expansão pulmonar de unidades em decorrência de atelectasias e colabamentos dos alvéolos (França et al., 2012; Associação de Medicina Intensiva Brasileira/Sociedade Brasileira de Pneumologia e Tisiologia [AMIB/SBPT], 2013). Além destas doenças, a PEEP também auxilia no tratamento de patologias como Doença Pulmonar Obstrutiva Crônica (DPOC), Síndrome Respiratória Aguda Grave (SARS), no qual atualmente destaca-se a Covid-19 (Ribeiro et al., 2021; Zhiling et al., 2021).

No entanto, a PEEP quando utilizada em altos níveis e longos períodos, há a possibilidade de desencadear repercussões hemodinâmicas, iniciadas a nível alveolar (Associação de Medicina Intensiva Brasileira/Sociedade Brasileira de Pneumologia e Tisiologia [AMIB/SBPT], 2013). Com a compressão dos capilares, devido a PEEP, há uma diminuição do retorno venoso e débito cardíaco, por consequência diminuição da pré-carga nos ventrículos direito e esquerdo, e aumento da pós-carga no ventrículo direito, gerando o aumento da pressão arterial, sendo nescessário ajustes autonômicos para reestabelecer a homeostasia circulatória (Azevedo et al., 2010; Vinhal, 2015). 
Para tanto, apenas em níveis entre 10, 15 e 20 cmH$_{2} \mathrm{O}$ são vistas repercussões hemodinâmicas (Associação de Medicina Intensiva Brasileira/Sociedade Brasileira de Pneumologia e Tisiologia [AMIB/SBPT], 2013; Azevedo et al., 2010; Barbas, 2013). No entanto estudos anteriores analisaram valores de PEEP em até $10 \mathrm{cmH}_{2} \mathrm{O}$, e não observaram repercussões hemodinâmicas e de balanço autonômico cardíaco (Louw, Médigue, Papelier, Cottin, 2010; Giustiniano et al, 2009; Choi, 2015; Kemerci, 2016; Sen \& Doventas, 2017).

Nesse contexto, ainda há níveis de PEEPs superiores aos já relatados, ainda não compreendidos em ensaios clinicos, que podem ampliar as possibilidades terapêuticas ou limita-las, podendo garantir maior segurança ao paciente bem como eficácia da abordagem.

O objetivo deste estudo foi avaliar o efeito da pressão positiva expiratória final sobre a hemodinâmica e balanço autonômico cardíaco de pacientes em uso de ventilação mecânica invasiva.

\section{Metodologia}

Trata-se de um ensaio clínico não probabilístico, onde os pacientes são controles deles próprios (Köche, 2011), realizado na Unidade de Terapia Intensiva (UTI) do Hospital Regional de Lagarto-SE, no período de janeiro de 2018 a julho de 2018. Os pacientes admitidos na UTI após 24 horas foram elegíveis para o estudo. Os excluídos foram pacientes com idade inferior a 18 anos, que apresentassem histórico de tabagismo ou etilismo, história de doenças cardíacas, doença arterial coronariana, doença renal, neurológica e metabólica.

A intervenção acorreu apenas em uma instância. Os pacientes foram mantidos em decúbito dorsal, posição de Fowler $\left(45^{\circ}\right)$, modalidade de ventilação em Pressão de Suporte Ventilatório (PSV) e parâmetro inicial de PEEP em $8 \mathrm{cmH}_{2} \mathrm{O}$. O cardiofrequencimetro (POLAR V800) foi envolto no toráx do paciente e centralizado no esterno, para inicio da coleta. Inicialmente por 15 minutos, foi coletado os dados em PEEP de $8 \mathrm{cmH}_{2} \mathrm{O}$, logo em seguida ajustado para $12 \mathrm{cmH}_{2} \mathrm{O}$ e coletado por mais 15 minutos, e por fim ajustado para $15 \mathrm{cmH}_{2} \mathrm{O}$ por 15 minutos finais, registrando parâmetros hemodinâmicos e balanço autonômico cardíaco.

As variáveis hemodinâmicas (frequência cardíaca, RH; pressão arterial sistólica, PAS; pressão de sangue diastólica, DBP; pressão arterial média, MAP; pressão de pulso, PP; e duplo produto, DP) foram colhidas durante a intervenção, nos 15 minutos sucessivos a cada incremento de PEEP $\left(8,12\right.$ e $\left.15 \mathrm{cmH}_{2} \mathrm{O}\right)$.

Com relação às variáveis hemodinâmicas da pesquisa, a FC foi verificada por um monitor multiparamétrico (Dash $4000^{\mathrm{TM}}$ Patient Monitor - GE Healthcare) conectada ao paciente através de eletrodos descartáveis no tórax. A PAM, PAD e PAS foram medidas através do esfigmomanômetro acoplado ao monitor.

Para às variáveis da variabilidade da frequência cardíaca (low frequency, LF; high frequency, HF; razão LF/HF) foi utilizado um Cardiofrequencímetro da marca POLAR modelo V800 envolto ao tórax do paciente.

Os dados foram apresentados por média e desvio padrão e para tratamento estatístico, foi utilizado o Graph Pad Prism 6.0. A distribuição dos dados foi avaliada pelo Shapiro - Wilk teste. As variáveis que apresentaram distribuição normal foram analisadas pelo teste de ANOVA de uma via com medidas repetidas e pós-teste de Tukey. Já para as variáveis não paramétricas foi realizado o teste de Friedman. Valores de $\mathrm{p}<0,05$ indicaram significância estatística.

Este estudo foi financiado pela FAPITEC / SE - Fundação de Amparo à Pesquisa e Inovação Tecnológica do Estado de Sergipe (Edital MS / CNPQ / FAPITEC / SE / SES nº 02/2017 PPSUS SE). E não houve conflitos de interesse para realização do presente estudo.

Foi apresentado o Termo de Consentimento Livre e Esclarecido (TCLE) aos responsáveis dos participantes da pesquisa, nele estava explícito os objetivos do estudo, bem como seus potenciais benefícios, ausência de danos e a garantia de da 
confidencialidade e sigilo das informações colhidas, cumprindo os princípios do rigor ético da Resolução 466/12 (Brasil, 2013). Este estudo foi aprovado pelo Comitê de Ética e Pesquisa com Seres Humanos (CEP) - CAAE (82329017.3.0000.5546), sob o parecer $n^{\circ}$ 2.537.641. Além disso, o ensaio clínico foi registrado como RBR28gq469 no Registro Brasileiro de Ensaios Clínicos (REBEC). Sendo assim, a pesquisa seguiu o que compete a resolução ética no 510 de 7 de abril de 2016 (Brasil, 2016).

\section{Resultados}

O estudo incluiu 30 pacientes submetidos sobre ventilação mecânica invasiva, em sua maioria homens 19 (63,33\%), seguido de 11 mulheres $(36,66 \%)$. Grande parte dos indivíduos $(\mathrm{n}=21 ; 70 \%)$ apresentavam faixa etária acima de 65 anos, seguido de 41 a 65 anos, com uma amostragem de 7 pacientes (23,33\%) e pôr fim a faixa etária de 18 a 40 anos totalizando 2 pacientes $(6,66 \%)$, tendo a amostra idade média de 71,37 $\pm 16,4$ anos.

Nenhuma complicação foi observada durante nosso protocolo, e a exclusão de pacientes não foi necessária. Nenhuma mudança foi encontrada nas variáveis hemodinâmicas (Tabela 1) e na variabilidade da frequência cardíaca (Figura 1) entre os pacientes submetidos aos níveis de PEEP apresentados.

Tabela 1. Variáveis hemodinâmicas de pacientes em ventilação mecânica submetidos aos níveis de PEEP.

\begin{tabular}{lcccc}
\hline \multirow{2}{*}{ Variáveis } & \multicolumn{4}{c}{ PEEP $\left(\mathbf{c m H} \mathbf{H}_{\mathbf{O}} \mathbf{)}\right.$} \\
\cline { 2 - 5 } & $\mathbf{8}$ & $\mathbf{1 2}$ & $\mathbf{1 5}$ & $\boldsymbol{p}$ \\
\hline HR (bpm) & $89,23 \pm 15,42$ & $88,70 \pm 13,10$ & $89,07 \pm 14,12$ & 0,96 \\
MAP (mmHg) & $93,22 \pm 13,87$ & $93,56 \pm 13,26$ & $94,82 \pm 13,37$ & 0,11 \\
SBP (mmHg) & $129,3 \pm 24,79$ & $128,3 \pm 23,18$ & $128,1 \pm 21,82$ & 0,94 \\
DBP (mmHg) & $75,17 \pm 11,52$ & $76,17 \pm 10,50$ & $78,17 \pm 12,68$ & 0,11 \\
PP (mmHg) & $54,17 \pm 21,31$ & $52,17 \pm 18,56$ & $49,93 \pm 19,77$ & 0,23 \\
DP (mmHg.bpm) & $11999 \pm 2631$ & $11369 \pm 2687$ & $11365 \pm 2419$ & 0,21 \\
\hline
\end{tabular}

PEEP: pressão expiratória final positiva; FC: frequência cardíaca; PAM: pressão arterial média; PAS: pressão arterial sistólica; PAD: pressão arterial diastólica; PP: pressão de pulso; DP: duplo produto. Fonte: Autores (2018). 
Figura 1. Variabilidade da Frequência Cardíaca nas Faixas Baixa Frequência, Alta Frequência e HF / LF de pacientes em ventilação mecânica submetidos a PEEP de 812 e 15. Ventilação mecânica submetida a PEEP de 812 e 15.

A

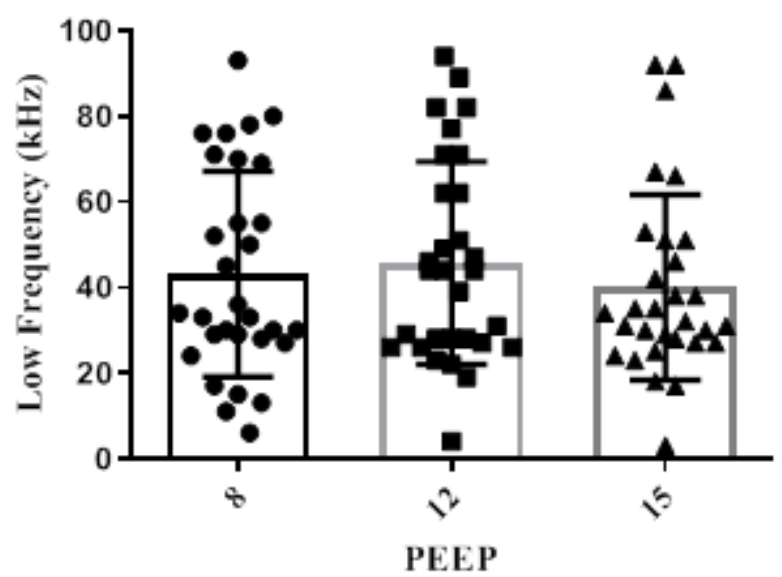

PEEP
B

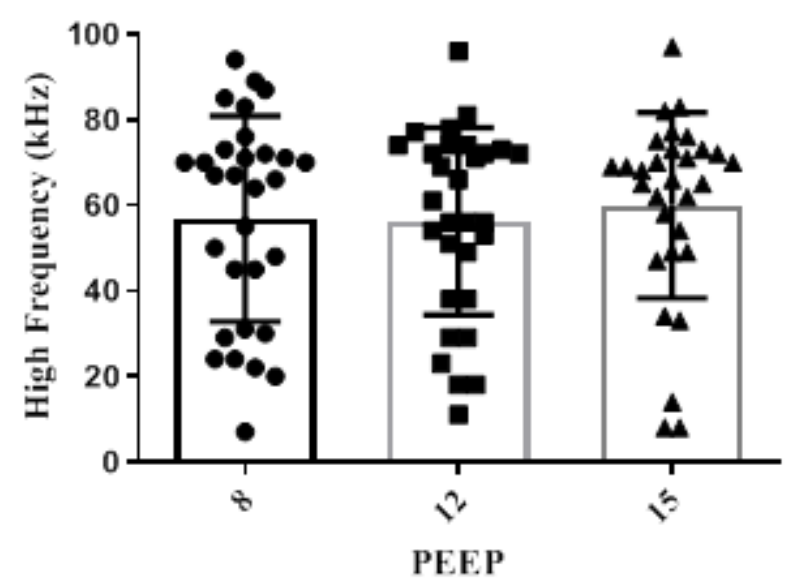

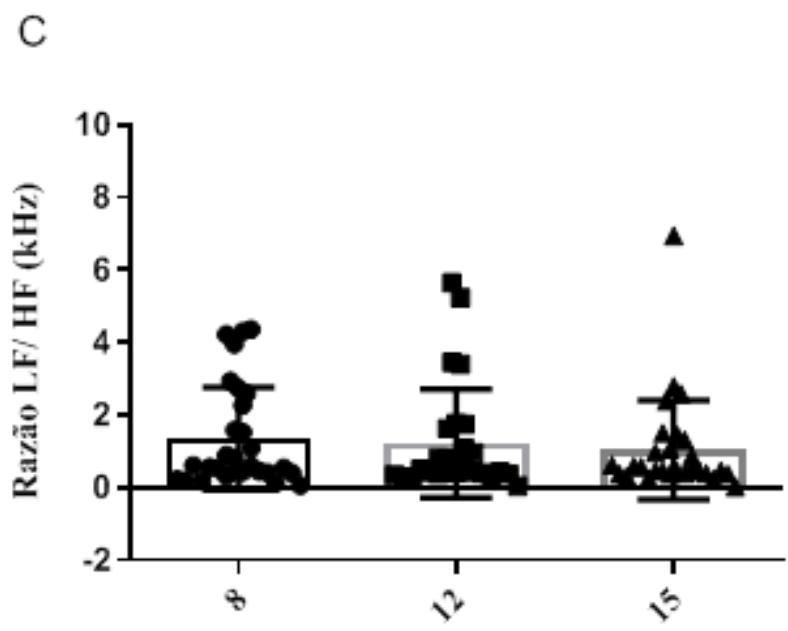

PEEP

Figura A.1: Variabilidade da Frequência Cardíaca nas Faixas Baixa Frequência, Alta Frequência e HF / LF de pacientes em ventilação mecânica submetidos à PEEP de 812 e 15. Para avaliar a diferença entre os valores da PEEP, foi utilizado o teste de Friedman. Os resultados foram expressos como média \pm desvio padrão. (p> 0,05). Fonte: Autores (2018).

\section{Discussão}

A principal conclusão deste estudo foi que os niveis de PEEP (8, 12 e 15 cmH2O) analisados não causaram mudanças na FC, PAS, PAD, PAM, PP, DP, LF, HF e razão LF/HF em pacientes ventilados mecanicamente.

Sobre uma perspectiva fisiologica, conforme alguns autores, seriam necessários valores de PEEP entre 10,15 e 20 cmH2O para gerar compressão dos capilares, diminuindo o retorno venoso e débito cardíaco respectivamente, alterando níveis pressóricos arteriais, levando a ajustes autonômico imediatos na macro-hemodinâmica (Associação de Medicina Intensiva Brasileira/Sociedade Brasileira de Pneumologia e Tissiologia [AMIB/SBPT], 2013; Azevedo et al., 2010; Vinhal, 2015; Pereira et al., 2018).

Diferentes estudos avaliaram a PEEP e suas repercussões hemodinâmicas e do balanço autonômico cardíaco em níveis de até $10 \mathrm{cmH} 2 \mathrm{O}$. Compararam níveis diferentes de PEEP (0, 5 e $10 \mathrm{cmH} 2 \mathrm{O})$ em pacientes submetidos a despinçamento da 
aorta, um estudo observou apenas uma limitação do aumento da PAM nos níveis de 5 e $10 \mathrm{cmH} 2 \mathrm{O}$, quando comparados PEEP de 0 (Giustiniano et al, 2009), diferente do nosso estudo que não foi possível evidenciar tal fato.

Em estudo com pacientes em colecistectomia laparoscópica observou-se que o uso da PEEP a $10 \mathrm{cmH} 2 \mathrm{O}$ resgatou a saturação de oxigênio cerebral, não obtendo efeitos adversos sobre a estabilidade hemodinâmica (Kwak, Park, Lee, Lee \& Kim,2013). Em estudo anterior foi observado efeitos da PEEP em pacientes durante a prostatectomia radical laparoscópica assistida, utilizando valores de PEEP de 0, 3, 5, 7 e 10 cmH2O onde não foi encontrado alterações hemodinâmicas (Karsten et al., 2011).

Em corroboração, estudos seguintes utilizando PEEPs de até $10 \mathrm{cmH} 20$ durante a cirurgias laparoscópicas, não levaram a alterações significativas quanto aos parâmetros hemodinâmicos avaliados (Choi, 2015; Kemerci, 2016; Sen \& Doventas, 2017), observando apenas o aumento da FC na PEEP $10 \mathrm{cmH} 2 \mathrm{O}$ quando comparado com PEEP $0 \mathrm{cmH} 2 \mathrm{O}$ (Kemerci, 2016).

Diferente dos demais, apenas o um estudo avaliou além da hemodinâmica, a sensibilidade barorreflexa em pacientes mecanicamente ventilados nos níveis de PEEP de 5 e $10 \mathrm{cmH2O}$. Em seu estudo foi possível identificar que apenas a PEEP de $10 \mathrm{cmH} 2 \mathrm{O}$ apresentou menor ganho de sensibilidade barorreflexa e amplitude de variação da HF-RR quando comparada a PEEP de $5 \mathrm{~cm} \mathrm{H2O}$ (Louw, Médigue, Papelier, Cottin, 2010), o que também não foi possível observar no nosso estudo, visto que a variabilidade da frequência cardíaca mantiveram-se homogêneas.

Por outro lado, em estudos recentes é possível constatar que os parâmetros macro-hemodinâmicos, podem ser dissociados da microcirculação, estando diretamente relacionadas a distribuição de fluxo sanguíneo local (Tafner et al., 2017). No estudo conduzido em pacientes na UTI com choque hemorrágico, demonstrou que mesmo na presença de parâmetros macrocirculatórios normais, a microcirculação sublingual encontrava-se disfuncional por até 3 dias após o choque (Tachon et al., 2014).

Ainda assim, os estudos apresentados em sua maioria não foram capazes de gerar alterações na macrocirculação entre os parâmetros hemodinâmicos avaliados (FC, PAM, PAS, PAD) e no balanço autonômico cardíaco diretamente ligadas à PEEP, quando sujeitos a níveis de pressão de até $10 \mathrm{cmH} 2 \mathrm{O}$.

Vale ainda ressaltar que os ensaios clínicos apresentados, apenas avaliaram níveis de até $10 \mathrm{cmH} 2 \mathrm{O}$. Em nosso

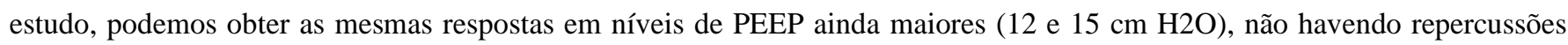
hemodinâmicas e de variabilidade da frequência cardíaca. Em contrapartida, novas lacunas abrem-se sobre os níveis acima de $15 \mathrm{cmH} 2 \mathrm{O}$, sendo sugerido repercussões hemodinâmicas entre 10 e $20 \mathrm{cmH} 2 \mathrm{O}$, os quais não foram encontrados estudos para tais condições (Associação de Medicina Intensiva Brasileira/Sociedade Brasileira de Pneumologia e Tisiologia [AMIB/SBPT], 2013; Azevedo et al., 2010; Vinhal, 2015).

\section{Conclusão}

No presente estudo, os niveis de PEEP $\left(8,12\right.$ e $\left.15 \mathrm{~cm} \mathrm{H}_{2} \mathrm{O}\right)$ não promoveram alterações nas respostas hemodinâmicas e no balanço autonômico cardíaco em pacientes sobre ventilação mecânica invasiva.

Este estudo foi limitado pela baixa rotatividade de pacientes. Outros níveis de PEEP devem ser testados, bem como diferentes tempos de aplicação em pacientes ventilados mecanicamente.

Concluindo, é importante enfatizar a importância do conhecimento sobre esta temática, pois, este trabalho demonstra uma vasta possibilidade para trabalhos futuros com pesquisas que abranjam melhor o perfil do paciente e/ou as técnicas utilizadas.

DPS, ASB, TCFC contribuiu para o desenho do estudo e discussão dos resultados; DPS, LKCO e GCS contribuíram para a coleta de dados; DPS e ASB contribuiu para a análise estatística e participou ativamente da redação das seções 
estatísticas do manuscrito; Todos os autores revisaram criticamente o manuscrito e aprovaram a versão final.

\section{Referências}

Araújo, J. N. M., Dutra, J. I. S., Silva, C. S. S., Macedo, H. K. S., Melo, J. J. S., Lopes, J. V. \& Medeiros, M. L. O. (2020). Orientações para o Uso da Ventilação Mecânica - Geral e em Pacientes Acometidos pela Covid-19. Santa Cruz - RN. https://wp-sites.info.ufrn.br/admin/wpcontent/uploads/sites/4/2020/05/Material_educativo_VM_Monitora_Media_e_Alta_complexidade_FACISA_UFRN.pdf

Associação de Medicina Intensiva Brasileira. Sociedade Brasileira de Pneumologia e Tisiologia (2013). Diretrizes Brasileira de Ventilação Mecânica. São Paulo: AMIB/SBPT.

Akker, J. P. C., Egal M. \& Groeneveld A. B. (2013). Invasive mechanical ventilation as a risk fator for acute kidney injury in the critically ill: a systematic review and meta-analysis. Revista Critical Care, 17 (3). https://www.ncbi.nlm.nih.gov/pmc/articles/PMC3706893/

Azevedo, J. C. M., Carvalho, E. R. M., Feijó, L. A., Oliveira, F. P., Menezes, S. L. S., \& Murad, H. (2010). Effects of the Continuous Positive Airway Pressure on the Airways of Patients with Chronic Heart Failure. Arquivos Brasileiro de Cardiologia, 95(1). https://www.scielo.br/j/abc/a/n4dBLCdftvR3y4fL6cpF7pb/?lang=en

Barbas, C. S., Ísola, A. M., Farias, A. M., Cavalcanti, A. B., Gama, A. M., Duarte, A. C. \& Amado, V. M. (2014). Recomendações brasileiras de ventilação mecânica 2013. Parte I. Revista Brasileira de Terapia Intensiva, 26(2), 89-121. https://www.scielo.br/j/rbti/a/Whwrm75h6MJwr5C6JmJg73Q/

Brasil. Ministério da Saúde. Conselho Nacional de Saúde. (2013). Resolução n 466, de 12 de dezembro de 2012. Ministério da Saúde.

Brasil. Ministério da Saúde. Conselho Nacional de Saúde. (2016). Resolução no 510 de 7 de abril de 2016. Ministério da Saúde.

Choi, Y. S., Bae, M. K., Kim, S. H., Park, J., Kim, S. Y., \& Oh, Y. J. (2015). Effects of Alveolar Recruitment and Positive End-Expiratory Pressure on Oxygenation during One-Lung Ventilation in the Supine Position. Yonsei Med Journal, 56(5), 1421-1427. https://eymj.org/DOIx.php?id=10.3349/ymj.2015.56.5.1421

Forgiarini, L. A. J., Rezende, J. C. \& Forgiarini, S. G. I. (2013). Manobra de recrutamento alveolar e suporte ventilatório perioperatório em pacientes obesos submetidos à cirurgia abdominal. Revista Brasileira de Terapia Intensiva, 25(4), 312-318. http://rbti.org.br/artigo/detalhes/0103507X-25-4-9

França, E. E. T., Ferrari, F., Fernandes, P., Cavalcanti, R., Duarte, A., Martinez, B. P. \& Damasceno, M. C. P. (2012). Physical therapy in critically ill adult patients: recommendations from the Brazilian Association of Intensive Care Medicine. Revista Brasileira de Terapia Intensiva, 24(1), 6-22. http://rbti.org.br/artigo/detalhes/0103507X-24-1-3

Giustiniano, E., Cancellieri, F., Battistini, G. M., Dominoni, C., Brancato, G., \& Spoto, M. R. (2009). Positive end-expiratory pressure during infrarenal aortic clamping limits hemodynamic impairment risk. Journal of Cardiovascular Medicine, 10(3), 282-287. https://europepmc.org/article/MED/19262219

Karsten, J., Luepschen, H., Grossherr, M., Bruch, H. P., Leonhardt, S., Gehring, H., \& Meier, G. T. (2011) Effect of PEEP on regional ventilation during laparoscopic surgery monitored by electrical impedance tomography. Acta Anaesthesiol Scand, 55(7), 878-886. https://onlinelibrary.wiley.com/doi/10.1111/j.1399-6576.2011.02467.x

Kemerci, P. U., Demir, A., Aydınl, B., Güçlü, Ç. Y., Karadeniz, Ü., Çiçek, Ö. F. \& Özgök, A. (2016). 10 cm H2O PEEP application in laparoscopic surgery and cerebral oxygenation: a comparative study with INVOS and FORESIGHT. Surg Endosc, 30, 971-978. doi: https://link.springer.com/article/10.1007/s00464-015-4277-8

Köche, J. C. (2011). Fundamentos de Metodologia Científica: Teoria da ciência e iniciação à pesquisa. Editora Vozes.

Kwak, H. J., Park, S. K., Lee, K. C., Lee, D. C. \& Kim, J. Y. (2013). High positive end-expiratory pressure preserves cerebral oxygen saturation during laparoscopic cholecystectomy under propofol anesthesia. Surg Endosc, 27, 415-420. https://link.springer.com/article/10.1007\%2Fs00464-012-2447-5

Louw, A. V., Médigue, C., Papelier, Y. \& Cottin, F. (2010). Positive end-expiratory pressure may alter breathing cardiovascular variability and baroreflex gain in mechanically ventilated patients. Respiratory Research, 26, 89-121. https://doaj.org/article/fb30a40f72114717b32388e 74773684c

Machado, F. D., Eder, G. L., Dulius, C. R., Baldisseroto, S. (2018). Ventilação Mecânica: Como Iniciar. https://docs.bvsalud.org/biblio ref/2018/04/882901/ventilacao-mecanica-como-iniciar.pdf

Pereira, S. M., Tucci, M. R., Morais, C. C. A., Simões, C. M., Tonelotto, B. F. F., Pompeo, M. S. \& Amato, M. B. P. (2018). Individual Positive Endexpiratory Pressure Settings Optimize Intraoperative Mechanical Ventilation and Reduce Postoperative Atelectasis. Anesthesiology, 129(6), 1070-1081. https://utsouthwestern.pure.elsevier.com/en/publications/individual-positive-end-expiratory-pressure-settings-optimize-int

Ribeiro, C., Vieira, A. L., Pamplona, P., Drummond, M., Seabra, B., Ferreira, D. \& Nunes, R. (2021). Práticas Atuais em Ventilação Mecânica Domiciliar para Doença Pulmonar Obstrutiva Crônica: Um Estudo Transversal Multicêntrico da Vida Real. Jornal Internacional de Doença Pulmonar Obstrutiva Crônica, 16, 2217-2226. https://www.dovepress.com/getfile.php?fileID=72156

Sem, O., Doventas, Y. E. (2017). Efeitos de diferentes níveis de pressão expiratória final sobre a hemodinâmica, mecânica respiratória e resposta sistêmica ao estresse durante colecistectomia laparoscópica. Campinas, SP: Revista Brasileira de Anestesiologia. https://www.scielo.br/j/rba/a/PYNHbxWJgYCBLymXmHc4wbn/abstract/?lang=pt

Vinhal, G. S. (2015). Efetitos hemodinâmicos da pressão positiva continua nas vias aéreas avaliados pelo ecodoppler cardiograma. (Dissertação de Mestrado). Universidade Federal de Uberlândia, Uberlândia. Brasil.

Tafner, P. F. A., Chen, F. K., Rabello, R. F., Corrêa, T. D., Chaves, R. C. F. \& Serpa, A. N. (2017) Recent advances in bedside microcirculation assessment in critically ill patients. Revista Brasileira de Terapia Intensiva, 29(2), 238-247. http://rbti.org.br/artigo/detalhes/0103507X-29-2-16 
Research, Society and Development, v. 10, n. 11, e336101119622, 2021

(CC BY 4.0) | ISSN 2525-3409 | DOI: http://dx.doi.org/10.33448/rsd-v10i11.19622

Tachon, G., Harrois, A., Tanaka, S., Kato, H., Huet, O., Pottecher, J. \& Duranteau, J. (2014). Microcirculatory Alterations in Traumatic Hemorrhagic Shock. Critical Care Medicine, 42(6), 1433-1441. https://neuro.unboundmedicine.com/medline/citation/24561562/Microcirculatory_alteratio ns_in_traumatic_hemorrhagic_shock_

Wang, T., Zhang, L., Luo, K., He, J., Ma, Y., Li, Z. \& Yu, X. (2016). Noninvasive versus invasive mechanical ventilation for immunocompromised patients with acute respiratory failure: a systematic review and meta-analysis. BMC Pulmonary Medicine, 16(1), 129. http://europepmc.org/article/PMC/5002326

Zhiling, Z., Hong, C., Qin, C., Nan, L., Shuisheng, Z., Qinggang, G. \& Gaiqi, M. (2021) Efeito da ventilação com pressão positiva não invasiva e oxigenoterapia nasal de alto fluxo na eficácia clínica de pacientes com nova pneumonia por coronavírus complicada por SDRA. Chinese Critical Care Medicine. 33(6), 708-713. http://rs.yiigle.com/CN121430202106/1331726.htm 